\title{
Reelin signalling in neuroblastoma: Migratory switch in metastatic stages
}

\author{
JÜRGEN BECKER ${ }^{1}$, JOHANNA FRÖHLICH ${ }^{1}$, CHRISTINA PERSKE ${ }^{2}$, \\ HELENA PAVLAKOVIC $^{3}$ and JÖRG WILTING ${ }^{1}$ \\ ${ }^{1}$ Center of Anatomy, Department of Anatomy and Cell Biology; ${ }^{2}$ Department of Pathology; \\ ${ }^{3}$ Center of Anatomy, Department of Neuroanatomy, University Medicine Goettingen, \\ Goettingen, Germany
}

Received January 19, 2012; Accepted March 8, 2012

DOI: $10.3892 /$ ijo.2012.1488

\begin{abstract}
The essential functions of Reelin for the migratory behaviour of neuroblasts in the central nervous system are well documented. Its role in the dissemination of neuronal tumours of the peripheral nervous system has not been studied in detail. Here, we examined neuroblastoma (NB), a tumour derived from sympathoadrenal cells of neural crest origin. We studied the expression of Reelin, its receptors VLDLR and LRP8 and the adapter protein DAB1 in primary tumour samples and cell lines. We used real-time RT-PCR, immunohistology and western blot analysis. In NB cell lines we studied effects of all-trans retinoic acid and the in vitro effects of Reelin. In primary tumour samples of untreated patients, a significant downregulation of Reelin and DAB1 mRNA was found in the metastatic stages 3, 4 and $4 \mathrm{~s}$. Immunohistochemical studies revealed expression of Reelin, LRP8 and DAB1 in differentiating-type low-grade NB In vitro, western blot analysis of selected NB cell lines showed variable expression patterns. Differentiation induction with all-trans retinoic acid induced the upregulation of Reelin and DAB1. Reelin acted as a chemoattractant for various NB cell lines but inhibited migration when applied together with the NB cells. In normal tissue, we found Reelin in lymphatic endothelial cells (LECs) but not in blood vessel endothelium (BECs). In primary NB, both BECs and LECs were positive. Our data strongly suggest that Reelin has a dual role in NB. Autocrine expression marks low-grade differentiating tumour cells, whereas paracrine Reelin presented by LECs and BECs may act as a chemoattractant and promote hematogenic and lymphogenic dissemination in progressed stages.
\end{abstract}

Correspondence to: Dr Jürgen Becker, Department of Anatomy and Cell Biology, University Medicine Goettingen, Kreuzbergring 36, D37075 Goettingen, Germany

E-mail: juergen.becker@med.uni-goettingen.de

Key words: neuroblastoma, reelin, very low density lipoprotein receptor, LRP8, Drosophila disabled homologue 1, lymphatics, tumor endothelial cells

\section{Introduction}

Neuroblastoma (NB) is a tumour of the neural crest-derived sympathetic nervous system and is therefore predominantly located in the paravertebral sympathetic trunk and in the adrenal medulla. It is the most frequent extracranial solid tumour in childhood with a peak of incidence in the second year of life. Clinically, NB is divided into stages 1, 2, 3, 4 and $4 \mathrm{~s}$, according to the international neuroblastoma staging system (INSS) (1-3). Roughly they form two distinct groups, which are characterized as follows. The first group, clinically referred to as stages 1 and 2, comprises localized and unilateral tumours with regional lymph node involvement only in stage $2 \mathrm{~B}$. The tumours can differentiate into benign ganglioneuroblastoma or even may regress spontaneously. The second group, stages 3 and 4, includes infiltrating and disseminating tumours with affection of loco-regional or contra-lateral lymph nodes in stage 3 and metastases to distant lymph nodes, bone marrow, liver and other organs in stage 4 . These lesions aggressively invade surrounding tissues, respond poorly to chemotherapy or relapse frequently due to the existence of therapy-resistant cells. While children with stage 1 and 2 tumours have a favourable prognosis, stage 4 NB often has an adverse outcome. The 'special' stage $4 \mathrm{~s}$, which, by definition, is only diagnosed in children younger than 1 year of age, bears features from both groups. It is characterised by skin and liver metastases and weak dissemination to lymph nodes and bone marrow. Despite the presence of metastases, these children have a favourable prognosis as their lesions respond well to mild chemotherapy and tend to have spontaneous differentiation or complete regression.

To date, the most important prognostic molecule in NB is the proto-oncogene $M Y C N$. Its amplification strongly correlates with failure of chemotherapy and unfavourable outcome (for review see refs. 4-6). We have started a search for molecules that control the metastatic behaviour of NB and observed regulation of the extra-cellular matrix protein keratoepithelin, the secreted hormone-like glycoprotein stanniocalcin-2 and the lymphangiogenesis inhibitor endogenous soluble vascular endothelial growth factor receptor-2 (esVEGFR-2) in disseminated stages of NB (7-10). At least in part, NB metastasizes along the lymphatic vascular system. In transcriptome micro-array 
analyses, we and others observed high expression of Reelin, a neuronal guidance molecule, in human lymphatic endothelial cells (LECs) as compared to blood vascular endothelial cells (BECs) (11-13). This prompted us to investigate the expression and function of Reelin in NB.

The Reelin gene was identified to harbour the mutation, which is causative for the phenotype of the so called 'reeler' mouse (14). Reelin encodes a secreted $388 \mathrm{kDa}$ extra-cellular matrix protein, which tends to form homodimers in vivo and may have serine-protease activity, although the latter has been called into question recently $(15,16)$. The spontaneous loss-offunction mutation of the Reelin gene in the reeler mouse strain causes inappropriate neuronal migration and lamination in the cerebral cortex and maldevelopment of the cerebellum, which leads to tremor and ataxia. Reelin is expressed in numerous tissues outside the CNS, but its functions there are practically unknown. Reelin signalling is predominantly transmitted by two trans-membrane receptors: the very low density lipoprotein receptor (VLDLR) and the apoprotein E receptor 2 (APOER2/ LRP8) (17). Binding of Reelin leads to clustering of the receptors and subsequent phosphorylation of DAB1 (Drosophila disabled homologue 1), an adapter protein associated with the intracellular domain of both receptors (18-20). Further downstream targets are members of the src-family and the protein kinase B/Akt pathway (21). Simultaneous knock-out of the receptors LRP8 (APOER2) and VLDLR, or disruption of DAB1, cause a phenotype highly similar to that of the Reeler mouse $(20,22)$. Besides VLDLR and LRP8, Reelin also binds to $\alpha 3 \beta 1$-integrin and thereby inhibits neuronal migration (23). Amyloid precursor protein (APP) is another potential receptor, located at synaptic membranes. It induces DAB1 phosphorylation upon Reelin binding and links Reelin to Alzheimer's disease (24). Recently, Yip et al have reported that Reelin and Dab1 are also expressed in the spinal cord and control the positioning of the preganglionic sympathetic neuron (25). Evangelisti et al observed regulation of Reelin by the microRNA-128 (miR-128) in NB (26). Since Reelin is a highly potent regulator of neuronal migration in the central nervous system, we hypothesized that it may be involved in the progression of NB. We show that Reelin induces migration of NB cells in vitro. Reelin is downregulated in metastatic NB stages, which may render the cells more susceptible for Reelin from other sources, e.g. the tumour LECs and BECs. We postulate that the downregulation of Reelin in tumour cells and its simultaneous upregulation in endothelial cells is part of a switch that promotes metastasis formation.

\section{Materials and methods}

Cell culture. Initially, neuroblastoma cell lines were cultured in RPMI-1640 medium containing 10\% fetal calf serum (FCS) and $1 \%$ penicillin/streptomycin (Lonza, Cologne, Germany). Cell supernatants for Reelin western blots were obtained from 48-h cultures in serum-free RPMI-1640. As we detected considerable amounts of Reelin in FCS (data not shown), we changed culture conditions for all subsequent experiments to serum-free PC1 medium (Lonza) and to serum-free RPMI1640 for migration assays. Neuroblastoma cell lines used in this study are: Kelly, Lan 2, NGP, SH-SY5Y, SK-N-AS and SMS-Kan. Commercial sources and references for all cell lines were published previously (8).
Supernatants from HEK-293 cells containing a Reelin expression plasmid or a control vector were obtained from 48-h cultures of nearly confluent plates with serum-free RPMI-1640 medium (17).

Human umbilical vein endothelial cells (HUVECs) were freshly prepared from umbilical cords and cultured in endothelial cell growth medium (Lonza). Lymphatic endothelial cells (LECs) were cultured in endothelial cell growth medium supplemented with VEGF-C and were characterized previously, as were the HUVECs (11).

Primary neuroblastoma samples and human tissues. RNA samples of 50 primary, untreated neuroblastomas, were provided by the German Neuroblastoma Studies Group (Drs F. Berthold, B. Hero and J. Theissen, Children's Hospital University Cologne, Cologne, Germany). The samples were tested for RNA integrity with a Bioanalyzer 2100 (Agilent Technologies, Böblingen, Germany). One sample failed the test and was discarded. The remaining 49 samples were of the following stages: stage 1 $(n=8)$, stage $2(n=6)$, stage $3(n=5 ; 2$ were MYCN-amplified), stage $4(n=20 ; 10$ were MYCN-amplified), stage $4 s(n=10 ; 1$ was MYCN-amplified).

Human foreskin was obtained from resection surgery at the University Medicine Goettingen. The studies were approved by the university's ethics committee.

Real-time RT-PCR. Reverse transcription was carried out with $1 \mu \mathrm{g}$ total RNA and Omniscript reverse transcriptase (Qiagen, Hilden, Germany) according to the manufacturer's instructions. For real-time RT-PCR we used the SYBR-Green JumpStart Taq ReadyMix (Sigma-Aldrich, Taufkirchen, Germany) and the following primer pairs (Iba, Göttingen, Germany): $\beta$-Actin_fwd 5'-GCATCCCCCAAAGTTCACAA-3', $\beta$-Actin_rev 5'-AGGA CTGGGCCATTCTCCTT-3', DAB1_fwd 5'-GCCTGGACAC ATTGACTGAA-3', DAB1_rev 5'-TCTTGCTGAGTGCAGT GTCC-3', LRP8_fwd 5'-CTGATGGCTCCGATGAGTC-3', LRP8_rev 5'-GGTCCACAGCTCAGCTTCTC-3', Reelin_fwd 5'-CATGGCTACAGCAACACACC-3', Reelin_rev 5'-GTGGG TGCACAGTGACATCT-3', VLDLR_fwd 5'-GGCAGTGTAA TGGTATCCGAGACT-3', VLDLR_rev 5'-AGGGCCCAAGC ACTGATTG-3'.

Western blot analysis. Cell culture supernatant of adherent cells was harvested and centrifuged for $10 \mathrm{~min}$ at $3,000 \mathrm{x} \mathrm{g}$ and $4^{\circ} \mathrm{C}$. Cells were counted and the volume of the supernatants was standardized to $1.5 \times 10^{6}$ cells. Cell lysates were prepared directly from cultured cells using lysis-buffer (30 mM Tris-Cl, pH 7.5, $150 \mathrm{mM}$ $\mathrm{NaCl}, 1 \mathrm{mM}$ EDTA, $1 \mathrm{mM}$ DTT) and sample complete protease inhibitor cocktail according to the manufacturer's instructions (Roche, Grenzach, Germany). Samples were adjusted to equal amounts of total protein, subjected to SDS-PAGE and subsequent protein blotting to a PVDF membrane (Roth, Karlsruhe, Germany). Membranes were blocked using 5\% bovine serum albumin in Tris-buffered saline (20 mM Tris-Cl, $150 \mathrm{mM} \mathrm{NaCl}$, $0.02 \%$ Tween-20) for 60 min. Primary and secondary antibodies were diluted in blocking buffer. Antibodies used were anti-Reelin (mouse monoclonal; Santa Cruz Biotechnology, Heidelberg, Germany), anti-VLDLR (mouse, monoclonal; Santa Cruz Biotechnology) anti- $\beta$-actin (mouse, monoclonal; Santa Cruz Biotechnology), anti-DAB1 (mouse monoclonal clonal; Abnova, 
Heidelberg, Germany), anti pY220 DAB1 (rabbit polyclonal; Abnova), anti-LRP8 (rabbit polyclonal; Abcam, Cambridge, UK). For detection we used horseradish peroxidase (HRP)coupled antibodies: goat anti-mouse HRP or goat anti-rabbit HRP (both Santa Cruz Biotechnology). Chemiluminescence was achieved using the Amersham ECL western blotting system (GE Healthcare) and detected and developed on SuperRX X-ray film (Fujifilm, Düsseldorf, Germany).

Immunohistology. Formalin-fixed primary NB samples were obtained from the German Neuroblastoma Studies Group (Children's Hospital University Cologne, Germany) and by the Department of Pathology, University Medicine Goettingen (Head: Professor H.J. Radzun). Histological grading was evaluated according to (27) and the International Neuroblastoma Pathology Classification (INPC), established in 1999 and modified in 2003 (28). Paraffin sections of $7 \mu \mathrm{m}$ were prepared for antigen retrieval as described recently (29). Primary antibodies used were anti-Reelin (mouse monoclonal; Santa Cruz Biotechnology), anti-LRP8 (rabbit polyclonal; Abcam), anti-DAB1 (goat polyclonal; Santa Cruz Biotechnology), antineurofilament (mouse monoclonal, Dako, Hamburg, Germany) and anti-Prox1 (rabbit polyclonal, Reliatech, Wolfenbüttel, Germany). Secondary antibodies were either peroxidaseconjugated goat-anti-rabbit IgG, rabbit anti-mouse IgG (1:100, Sigma-Aldrich) or Alexa 488-conjugated goat anti-mouse IgG, and Alexa 594-conjugated goat anti-rabbit IgG (both 1:200, Molecular Probes, Karlsruhe, Germany) for double immunofluorescence. DAB was used as chromogen for peroxidase reaction, and slides were counter-stained with nuclear fast red. For immunofluorescence nuclei were routinely stained with DAPI.

Induction of differentiation. SH-SY5Y $\left(10^{6}\right)$ were seeded on 10-cm cell-culture dishes at day 1 in serum-free PC1 medium (Lonza). They were allowed to adhere overnight. The next day (day 0) we started treatment with all-trans-retinoic acid (ATRA) dissolved in cell culture grade DMSO (Sigma). ATRA in DMSO was added to the medium to a final concentration of 5 and $10 \mu \mathrm{M}$. In control samples, the corresponding amount of DMSO was applied. At days 3,6 and 9 the medium was renewed and fresh ATRA or DMSO was added. RNA was isolated at days $0,5,9$ and 12 .

Migration assay. BD-Biocoat culture inserts (BD, Heidelberg, Germany) without any coating were used for the trans-well migration assays. Cells $(100,000)$ diluted in $0.5 \mathrm{ml}$ serum-free RPMI-1640 medium were seeded into the culture insert. The lower chamber was filled with $0.5 \mathrm{ml}$ culture supernatant of HEK-293 cells transfected with a Reelin expression plasmid (17) or the control vector. In another set of experiments, cells were suspended in supernatants of the Reelin transfected HEK-293 cells and migration towards RPMI-1640 containing $2.5 \%$ FCS as an attractant was studied. Supernatants were concentrated 2-fold using Vivaspin2 filter units (MWCO: 100 kDa; SartoriusStedim, Goettingen, Germany). After $24 \mathrm{~h}$, cells that migrated through the membrane towards the attractants were stained using Richardson's staining solution for $1 \mathrm{~min}$, mounted for microscopy and counted manually. Experiments were repeated at least three times. Percent of migrated cells compared to controls $(100 \%)$ were calculated.
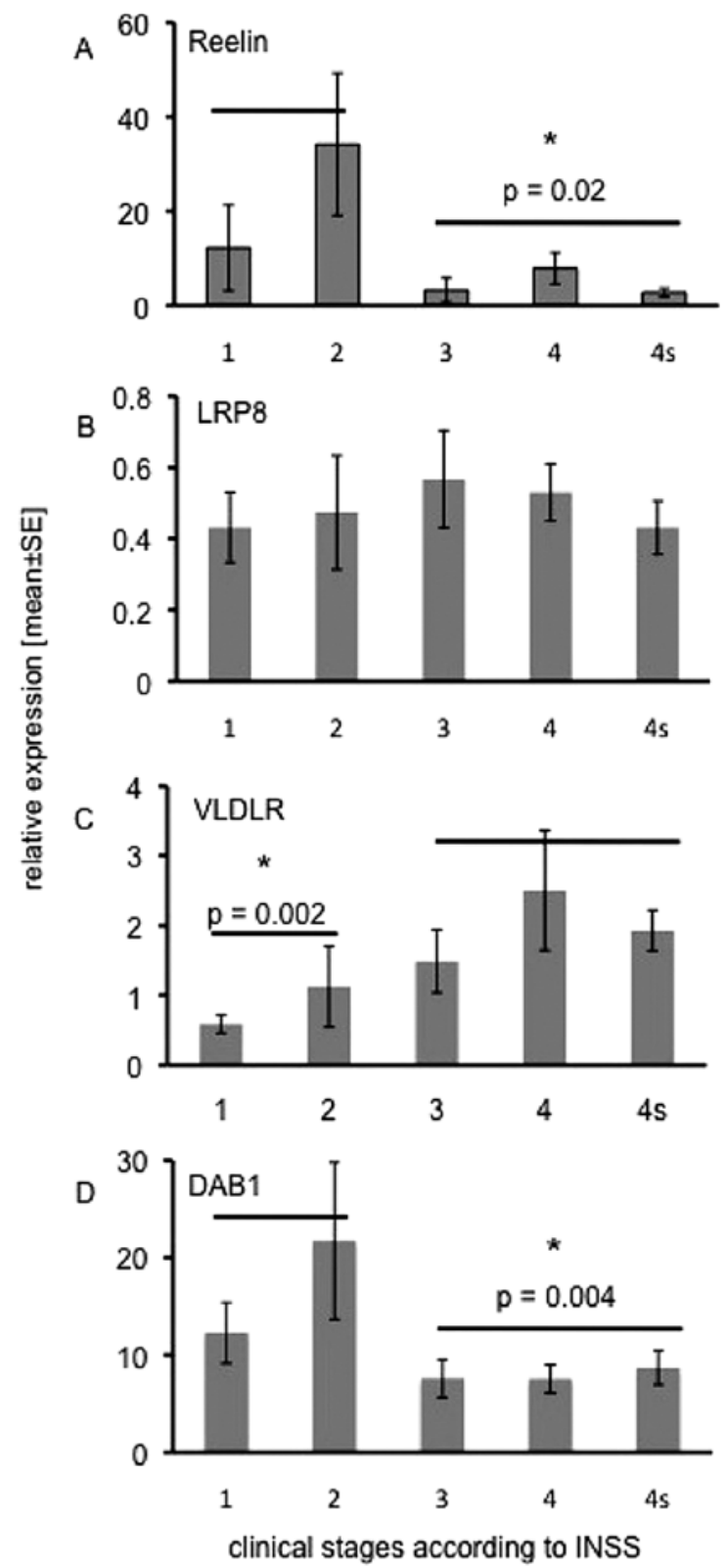

Figure 1. Expression of Reelin, LRP8, VLDLR and DAB1 in primary neuroblastoma samples. Neuroblastoma samples from all clinical stages (1-4 and 4s) were analysed by real-time RT-PCR. Mean relative expression and standard error of the mean are shown for each stage. (A) Note downregulation of Reelin mRNA in metastasized stages 3, 4 and 4s. (B) LRP8 is almost equally expressed in all stages. (C) VLDLR is upregulated in stages 3,4 and 4s. (D) DAB1 downregulation in metastasized stages 3, 4 and 4s. Vertical lines subsume stages that differ significantly. ${ }^{*}$ indicates statistical significance.

Calculations and statistics. Molecular weight of LRP8 and VLDLR were calculated from amino acid sequences provided by the NIH at www.ncbi.nlm.nih.gov using the pI-tool at www. expasy.org. For real-time RT-PCR analyses, relative expression levels of transcripts were calculated by the $\Delta \Delta \mathrm{Ct}$-method. Statistical analyses were performed using the GraphPad Prism v 3.0 software (GraphPad Software Inc., La Jolla, CA, USA) and Microsoft Excel 2008 for Mac (Microsoft Corp., Redmond, WA, USA). Normality was tested to calculate the variance distribution and the Mann-Whitney U test or the Unpaired t-test were used to compare expression levels between stages of localized 



Figure 2. Immunohistochemistry of primary neuroblastoma specimens. Staining of paraffin sections using antigen retrieval methods. (A, C and D) Anti-Reelin (B) negative control, (E) anti-LRP8 and (F) anti-DAB1. Sections were counter-stained using nuclear-fast-red. (A) Stage 3, grade 2 neuroblastoma. Note Reelin expression in tumour cells and in some cells in the stroma (*). (C) Stage 2, grade 3 neuroblastoma. Reelin is not detectable in the tumour cells. (D) Stage 1, grade 1 neuroblastoma. Note Reelin expression in differentiating neuroblasts. (E) Same specimen as in (A) showing LRP8 in tumour cells. (F) Same specimen as in (A) showing DAB1 in tumour cells. Bars, $60 \mu \mathrm{m}$ in (A-D) and $40 \mu \mathrm{m}$ in (E and F).

and disseminated NB and to compare expression levels in NB with or without MYCN amplification. Statistical significance is considered at $\mathrm{p}<0.05$.

\section{Results}

Expression of Reelin pathway molecules in primary $N B$. To investigate the potential clinical relevance of Reelin signalling, we measured the mRNA expression of Reelin and its potential signal transducers LRP8, VLDLR and DAB1 in 49 samples of untreated primary neuroblastoma patients. By real-time RT-PCR we found that Reelin transcripts were significantly more abundant in localized stage 1 and stage 2 tumours $(n=14)$ than in the metastatic stages 3,4 and $4 \mathrm{~s}(\mathrm{n}=35, \mathrm{p}=0.02$; Fig. 1A). The Reelin receptor LRP8 (Fig. 1B) was equally expressed in all stages, whereas the expression of the second Reelin receptor, VLDLR, continuously increased from stage 1 to stage 4 (Fig. 1C). Statistical analyses revealed that VLDLR expression levels were significantly lower in stages 1 and 2 vs. stages 3, 4 and $4 \mathrm{~s}(\mathrm{p}=0.002)$. Like Reelin, the adapter protein DAB1 (Fig. 1D) was significantly higher in stage 1 and stage 2 tumours as compared to the disseminated stages 3,4 and $4 \mathrm{~s}(\mathrm{p}=0.004)$.

We then studied immunolocalization of Reelin signalling molecules in primary NB samples. We observed variable immunoreactivity intensities for Reelin, LRP8 and DAB1 in NB samples. Thereby, expression of Reelin was higher in low-grade tumours, where we observed signals in NB cells that showed signs of differentiation (Fig. 2A, B and D). In undifferentiated grade 3 NB, Reelin was almost absent (Fig. 2C). Reelin was also detectable, though at a lesser extent, in some stroma cells (marked by an asterisk in Fig. 2A). In normal tissues LRP8 was located in the cell membrane (data not shown), whereas in NB it was mainly present in the cytoplasm (Fig. 2E). For VLDLR we could not find appropriate antibodies that complied with the paraffin-embedded specimens. DAB1 was found in the cytoplasm of differentiating type NB cells (Fig. 2F). 


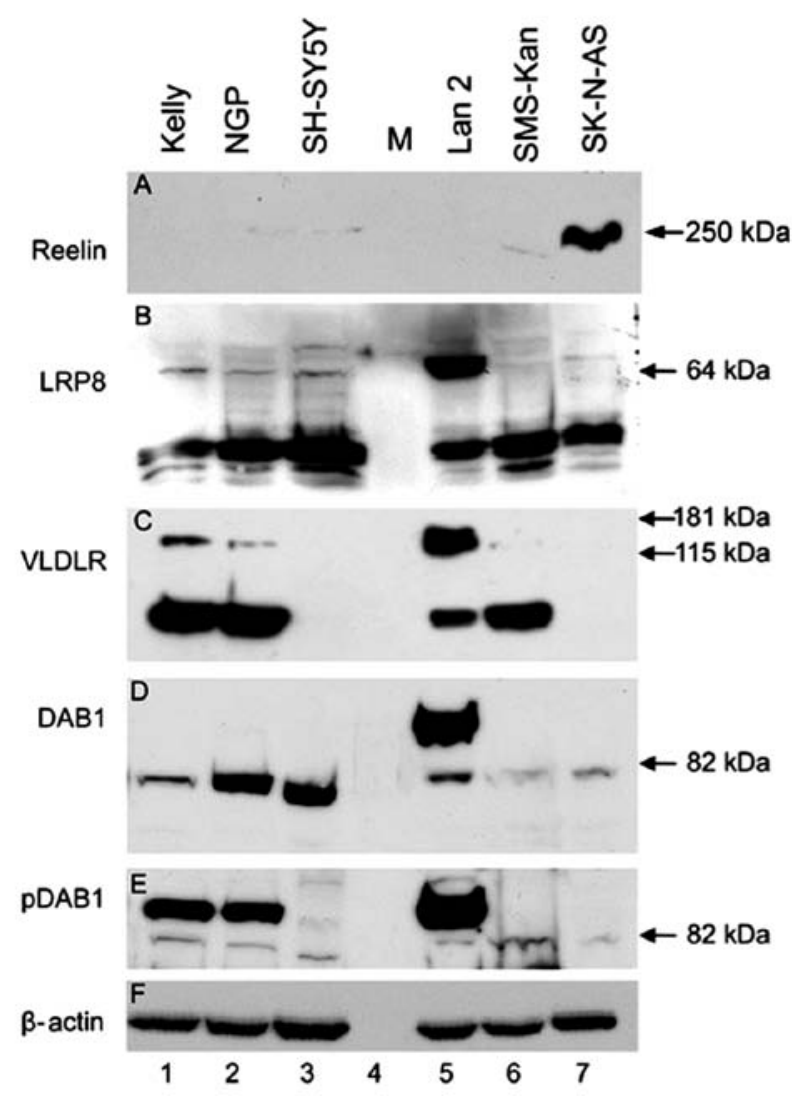

Figure 3. Protein expression of LRP8, VLDLR and DAB1 in selected cell lines. Western blotting was performed using cell lysates or culture supernatant of the cell lines Kelly (lane 1), NGP (lane 2), SH-SY5Y (lane 3), Lan 2 (lane 5), SMS-Kan (lane 6) and SK-N-AS (lane 7). Size marker (M) was loaded in lane 4. (A) Reelin, (B) LRP8, (C) VLDLR, (D) DAB1, (E) phospho tyr220 DAB1, (F) $\beta$-actin immunoreactivity is shown using antibodies as described in Materials and methods. Note that for each protein the specific bands appear but additional bands in some cell lines indicate altered or pathologic processing. The anti- $\beta$-actin was used as loading control and shows a regular band in all cell lines.

Expression in NB cell lines. For a further evaluation of Reelin signalling molecules, we screened 24 NB cell lines by real-time RT-PCR and found varying levels of Reelin, LRP8, VLDLR and DAB1 (data not shown). We then selected 6 cell lines for western blot analyses of whole cell lysates and, in case of Reelin, supernatants of these cell lines. In Reelin-transfected HEK-293 cells we found three Reelin bands at 400, 250 and $180 \mathrm{kDa}$ (data not shown), as described previously (17). Reelin was only present in the supernatant of SK-N-AS (Fig. 3A), which may be due to the fact that NB cell lines have usually been isolated from progressed NB. We observed a major band at $250 \mathrm{kDa}$. For LRP8, which was consistently found at the RNA level, we could detect a major band at approximately $35 \mathrm{kDa}$ in Kelly, NGP, SH-SY5Y, Lan 2, SMS-Kan and SK-N-AS (Fig. 3B). However, in Lan 2 we detected a second band at $60 \mathrm{kDa}$.

For VLDLR (Fig. 3C) a major $64 \mathrm{kDa}$ band was detectable in Kelly, NGP, Lan 2 and SMS-Kan. In lysates of SH-SY5Y and SK-N-AS no immunoreactivity for VLDLR was detected. Again, Lan 2 displayed a second major band at approximately $150 \mathrm{kDa}$, and unless much weaker, bands of the same molecular weight were also visible in Kelly and NGP cell lysates.
For DAB1 (Fig. 3D), there were two major immunoreactivity bands at approximately 115 and $82 \mathrm{kDa}$. All cell lines displayed the $82 \mathrm{kDa}$ band, albeit it was weak for Kelly, Lan 2, SMS-Kan and SK-N-AS. In SH-SY5Y lysates, however, the corresponding band had a slightly lower molecular weight. A second band at $115 \mathrm{kDa}$ was only found in Lan 2 cells. Constitutive DAB1 phosphorylation (pDAB1) was detected in Kelly, NGP and Lan 2 cells (Fig. 3E). For these experiments, cells were cultured in standard RPMI-1640 medium containing 10\% FCS. As we noticed later, FCS contains large amounts of Reelin (data not shown), probably leading to constitutive DAB1 phosphorylation. Migration and differentiation studies performed later were done with cells cultured under serum-free conditions using PC1 medium or basal RPMI-1640 medium. All blots were probed for $\beta$-actin to ensure equal loading (Fig. 3F).

Reelin is induced during differentiation of the NB cell line $S H-S Y 5 Y$. The expression of Reelin in the patients' samples suggested that Reelin expression might be associated with more differentiated tumour types and downregulated in undifferentiated high-grade tumours. It has been reported that Reelin is induced in the human teratocarcinoma cell line NT2 upon treatment with the differentiating agent all-trans retinoic acid (ATRA) (30), but downregulated in the NB cell line SH-SY5Y (26). ATRA is clinically used in NB treatment regimens, therefore we sought to test the response to ATRA with regard to Reelin and its signalling pathway molecules. For this study we also used the NB cell line SH-SY5Y, which is known to respond well to differentiating agents (31). By quantitative real-time RT-PCR we found that during 12 days of treatment with 5 or $10 \mu \mathrm{M}$ ATRA, Reelin, VLDLR and DAB1 mRNA levels increased significantly (Fig. 4A, C and D), whereas LRP8 mRNA expression decreased by $50 \%$ during the first 5 days and remained constant thereafter (Fig. 4B). Control experiments were performed with the solvent DMSO. For Reelin and DAB1 these findings correlate well with our observation that these molecules are significantly higher expressed in the NB stages 1 and 2 .

Reelin acts as a chemo-attractant on NB cells in vitro. Reelin is a key regulator in brain development and influences neuronal migration and patterning during cortex lamination. We tested whether Reelin also influences NB cell migration and subjected the NB cell lines SMS-Kan, LAN 2, SH-SY5Y, Kelly and SK-N-AS to trans-well migration assays (Fig. 5A). We used modified Boyden chambers and the supernatant of Reelinexpressing or control plasmid-expressing HEK-293 cells as an attractant. All tested cell lines showed increased migration towards the Reelin-containing medium. After $24 \mathrm{~h}$ the number of migrated cells increased by 2-fold for LAN 2 to nearly 7-fold for SK-N-AS as compared to the respective controls. We then tested if this migratory effect can be inhibited by Reelin. We incubated SK-N-AS cells in the upper chamber in 2-fold concentrated supernatant of the HEK-293 cells (Reelin-expressing vs. control) and observed a reduction of migration into the serumcontaining lower chamber by $60 \%$ (Fig. 5B).

Reelin is expressed in tumour endothelial cells. The downregulation of Reelin in advanced NB stages and the attractive potential of Reelin on NB cell lines suggests that additional Reelin sources may influence the metastatic behaviour of NB 

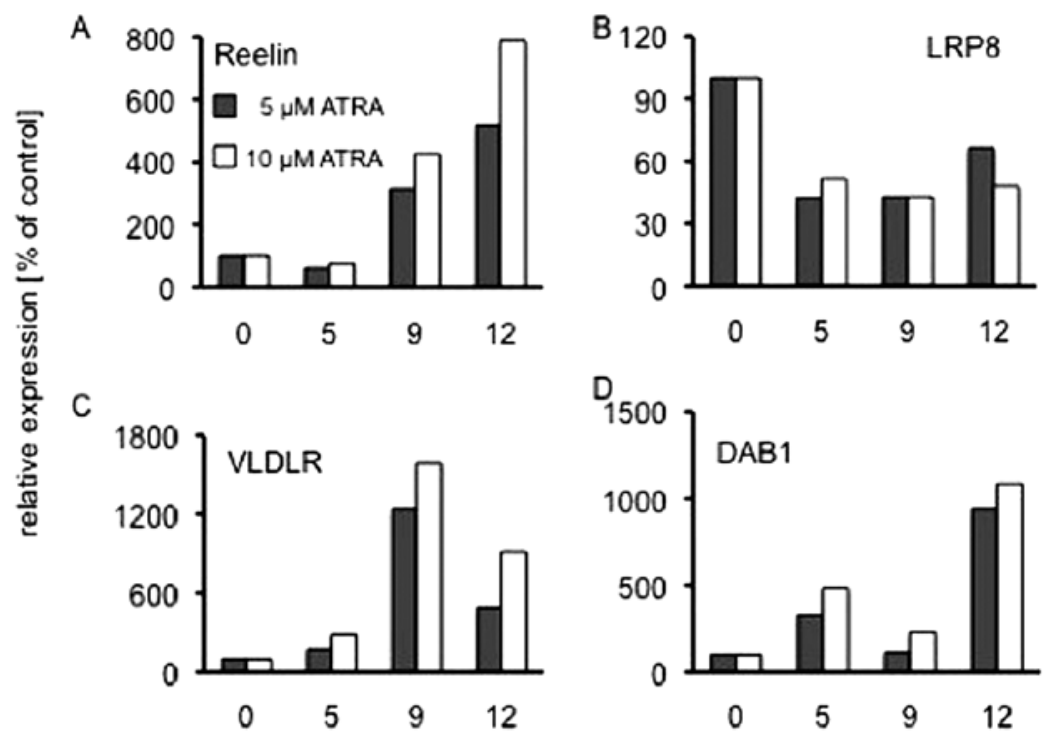

Figure 4. Differentiation of the cell line SH-SY5Y with ATRA. Cells were treated with 5 or $10 \mu \mathrm{M}$ ATRA for 0,5, 9 and 12 days. At indicated time points RNA was isolated and real-time RT-PCR performed. Bars indicate mean relative expression levels as percentage of DMSO controls. (A) Reelin expression is strongly upregulated at days 9 and 12. (B) LRP8 transcript levels decrease by $50 \%$ at day 5 , and remain stable afterwards. (C) VLDLR and (D) DAB1 levels increase during ATRA treatment.

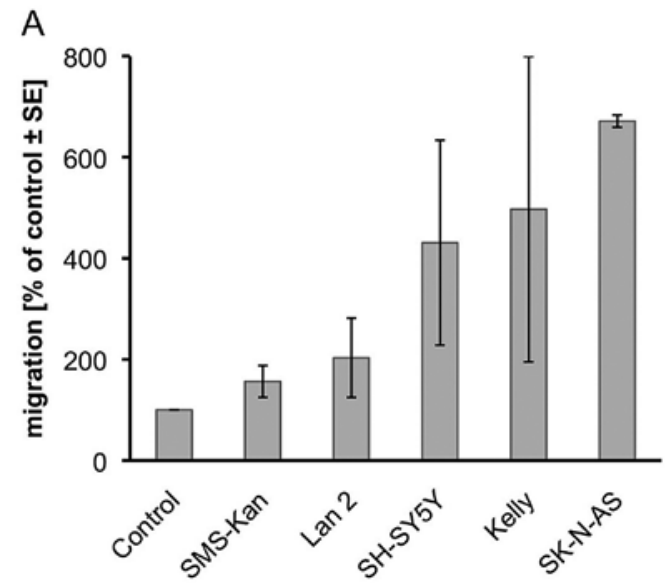

B

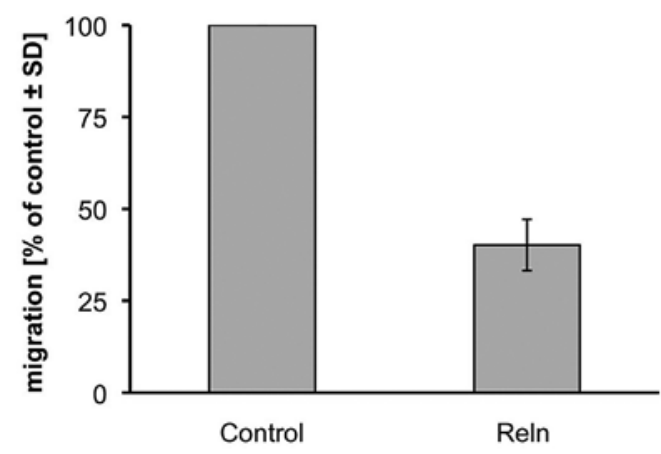

Figure 5. Reelin regulates NB cell migration. (A) In a trans-well assay, the NB cell lines SMS-Kan, LAN2, SH-SY5Y, Kelly and SK-N-AS were tested for their migration towards cell-supernatant of Reelin-expressing HEK-293 cells vs. control-transfected cells. Note induction of migration by Reelin. (B) SK-N-AS were pre-incubated with either supernatant of the Reelin-expressing HEK-293 cells or the control-transfected cells and tested for their migration towards a serum gradient. Pre-incubation with Reelin inhibits migration of the cells. The number of migrated cells is calculated as percentage of the respective contro experiments (control, 100\%). Means were calculated from at least three independent experiments with two replicates. cells. Previously, the mRNA expression of Reelin in normal lymphatic endothelial cells (LEC) has been found in microarray studies (11-13). At protein level, we detected the $250 \mathrm{kDa}$ form of Reelin as the dominant form in LECs, but no expression in HUVECs (Fig. 6A). By immunostaining with antibodies against the LEC marker Prox1 (32), we could demonstrate the presence of Prox1-positive lymphatics in primary NB (Fig. 6B), in accordance with previous studies (33). Immunoreactivity for Reelin was found in normal Prox1-positive initial lymphatics of human foreskin (Fig. 6C and D), and in lymphatic collectors (data not shown). In primary NB samples we found Reelin not only in lymphatics, but also in arteries and veins (Fig. 6E). Since Reelin expression is hardly detectable in normal BECs (11-13), this indicates upregulation of Reelin in tumour BECs.

\section{Discussion}

Reelin in primary neuroblastoma. Since the first description of the Reeler mouse some 60 years ago, the functions of Reelin have been studied intensively in the central nervous system (34). Abnormal lamination of the cerebral cortex, hypoplasia of the cerebellum and abnormal positioning of sympathetic neurons in the spinal cord have been attributed to impaired Reelin signalling $(25,35)$. In humans, malfunctions of Reelin are associated with neurological disorders such as schizophrenia, bipolar disorders, major depression, autism and lissencephaly (35), as well as lymphedema (36). Functions for Reelin in the peripheral nervous system (PNS), chromaffine cells and in tumours derived from the PNS, such as neuroblastoma (NB), have been studied very rarely (26,37-39). This encouraged us to investigate the expression patterns of Reelin, its receptors LRP8 and VLDLR, as well as DAB1, one of its most prominent downstream signalling molecules, in primary NB. Our real-time RT-PCR analyses of 49 primary samples of untreated patients reveal that the amount of Reelin and DAB1 transcripts are significantly lower in the advanced NB stages 3 and 4, and in 4s NB. While LRP8 


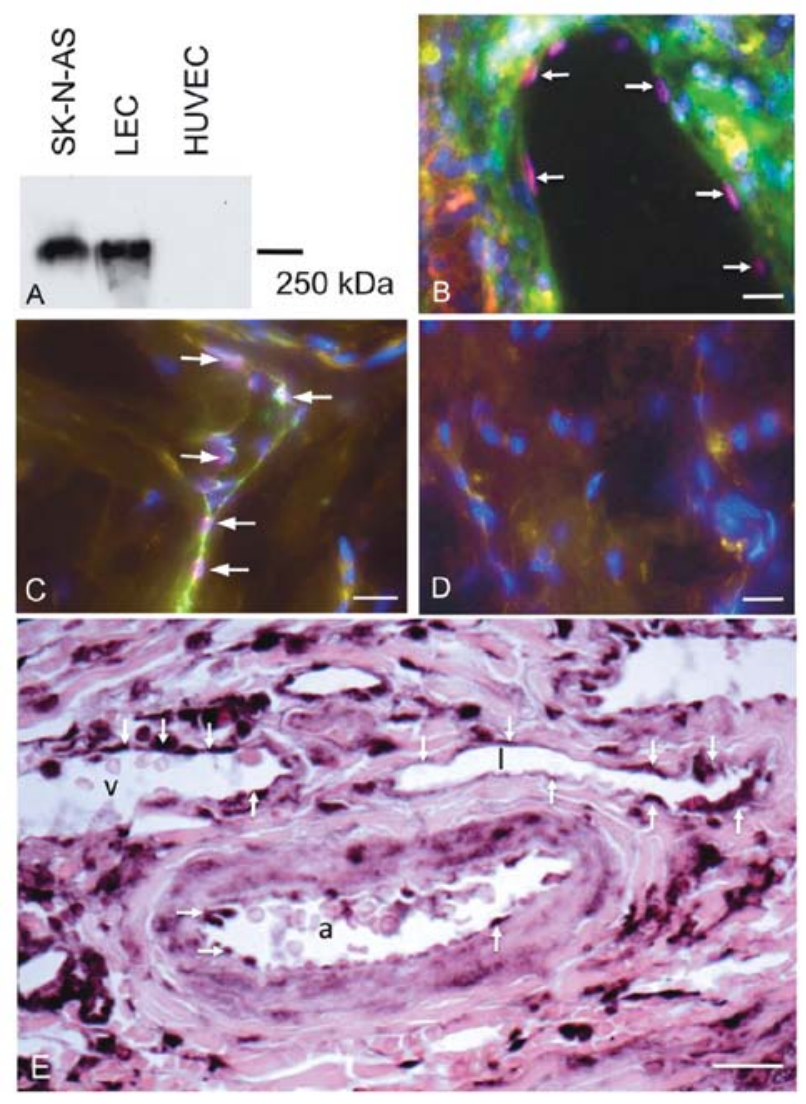

Figure 6. Vessels in neuroblastoma are Reelin-positive. (A) Western blot showing Reelin in SK-N-AS and in isolated LECs, but not in HUVECs (B) Primary NB were stained for Prox 1 expression to detect lymphatics. Immunofluorescence detection of Prox1 (red) in nuclei of LECs (arrows) and neurofilament in NB cells (green). (C) Co-expression of Reelin (green) and Prox1 (red nuclei, arrows) in lymphatics of normal human foreskin (D) Negative control of the specimen in (C). Nuclei are stained with DAPI (blue). (E) Peroxidase staining of Reelin in primary NB. Note expression in endothelial cells (arrows) of arteries (a), veins (v) and lymphatics (l). Bars, $20 \mu \mathrm{m}$ in (A and B) and $15 \mu \mathrm{m}$ in (C-E).

is not altered throughout the five stages, VLDLR expression is, in contrast, higher in the advanced stages, and in stage 4s. By immunohistochemistry we were able to confirm that Reelin, LRP8 and DAB1 are expressed by NB cells of primary tumours. Thereby, Reelin is mainly found in differentiating neuroblasts of low-grade tumours. Reelin is also detectable in the vascularised stroma of the tumours. Both tumour LECs and BECs express Reelin, whereas in normal tissues and endothelial cell lines we found Reelin protein in LECs but not in BECs, which is in line with transcriptome analyses performed in several labs (11-13). The results show that two key regulators of the Reelin signalling pathway, Reelin and Dab1, are significantly lower in progressed NB. Reelin is obviously a marker for differentiating and differentiated NB cells. It is almost absent in high-grade NB cells, but expressed in tumour LECs and upregulated in tumour BECs. Our results indicate that there is a switch from an autocrine function of Reelin in low-grade NB to a paracrine function in high-grade NB.

Reelin pathway molecules in NB cell lines. We screened 24 human NB cell lines for the mRNA expression of the Reelin pathway molecules. The complete list of cells can be found in (8). Using real-time RT-PCR we were able to detect transcripts of all four major molecules of Reelin signalling: Reelin, LRP8, VLDLR and DAB1. However, expression levels vary considerably between cell lines (data not shown). We chose 6 cell lines for western blot analyses and found differential expression of all four molecules, as well as the activated, phosphorylated form of DAB1, pDAB1. The pattern of bands appears to be more complex than in non-tumour cells. Secreted Reelin is only detectable in SK-N-AS supernatants. The other cell lines are negative, which may reflect the downregulation of Reelin in the advanced tumour stages that have been used for the isolation of the cell lines.

For LRP8, Kim et al have reported a molecular weight (MW) of $105 \mathrm{kDa}$. As estimated by sequence analysis, its MW should be in the range of $80-100 \mathrm{kDa}(40)$. However, none of our cell lines displayed a band for LRP8 in this range, but a strong signal at approximately $30 \mathrm{kDa}$. In murine neuronal cultures, the presence of a $30 \mathrm{kDa}$ band has been described recently, and the authors show that this represents a soluble splice variant of LRP8, which binds Reelin and prevents DAB1 phosphorylation (41). This suggests that in our NB cell lines there is no transmembrane LRP8, but rather an inhibitory soluble LRP8 variant. This may account for the observed immunolocalisation in the cytoplasm. A second band at $65 \mathrm{kDa}$, only present in Lan 2 cells, may also be due to alternative splicing, as it is known that several species- and tissue-specific splice variants of LRP8 do exist (42-44).

The second receptor for Reelin, VLDLR, is absent in SH-SY5Y and SK-N-AS. The other cell lines display a major band at $70 \mathrm{kDa}$. A second band at $150 \mathrm{kDa}$ is prominent in Lan 2, faintly detectable in Kelly and NGP, but absent in SMS-Kan. As the calculated size of VLDLR is $96 \mathrm{kDa}$ these bands may also be splice variants or isoforms of the gene. However, the signals we obtained are highly reproducible and must therefore be regarded as specific.

The activity of the adapter protein DAB1 is regulated by phosphorylation, and transcriptional quantities may not necessarily reflect its importance in the signalling pathway. For DAB1, we observed a major band at approximately $82 \mathrm{kDa}$ in all cell lines. A second band at $115 \mathrm{kDa}$ was present only in Lan 2 . When isolated from mouse brain, a band for Dab1 has been detected at $80 \mathrm{kDa}(45)$. We have not further analysed the $115 \mathrm{kDa}$ band in Lan 2, however it seems possible that this is caused by genetic instability. DAB1 is located on chromosome 1p, where genetic mutations, translocations and amplifications occur frequently in NB (6). We observed a $85 \mathrm{kDa}$ band of the activated pDAB1 only in Kelly, NGP, and Lan 2. We suggest that activation is due to the presence of Reelin in FCS, which we observed in immunoblot assays (data not shown). In order to prevent unwanted Reelin effects, we changed the culture conditions for our functional experiments and used serum-free media.

The variable patterns of Reelin signalling molecules in NB cell lines obviously reflect the origin of the cells, namely aggressive tumours and metastatic lesions (46). Roughly, low stage NB and more differentiated lesions have a better prognosis than advanced NB, consisting predominantly of undifferentiated 'small round' cells. We found that high Reelin expression correlates with both low staging and grading of the tumours. We therefore investigated Reelin expression after treatment with ATRA, a well-known differentiating agent in NB that is routinely used in clinical regimens. In accordance 
with earlier studies (30), we found that Reelin is induced upon this treatment. Thereby, the upregulation of Reelin takes time and can only be observed after 9 and 12 days of treatment, not after 5 days. In a comparable study, Evangelisti et al (26) found downregulation of Reelin after 6 days of ATRA treatment of SH-SY5Y. They did not study later time points. They used fetal bovine serum, which according to our experience contains Reelin. Unfortunately, they do not comment on the expression of Reelin in their 28 primary NB samples. Our data suggest that the expression of Reelin is a feature of late maturing sympathetic cells. However, expression of Reelin during development of the peripheral sympathetic nervous system and the adrenal medulla have not been studied in detail.

Guidance functions of Reelin. There is an ongoing debate whether Reelin acts as a stop-signal or as an attractant for neurons during patterning of the cerebral cortex (47). The high Reelin expression in low stage NB suggested a metastases inhibiting function, and we speculated it might act as a stop signal for NB cells. This speculation is strengthened by our results, showing that preincubation of SK-N-AS cells with Reelin-containing cell supernatant reduces the migration towards a serum gradient. However, we also demonstrate here that Reelin has migration-promoting abilities in all cell lines tested when it is used as an attractant. As discussed below, it appears that in vivo external sources provide attractive Reelin in a paracrine manner. The attraction of neurons by Reelin from Cajal-Retzius neurons has been shown earlier (48), but still it remains to be studied, how the signal is transmitted. Especially, SH-SY5Y and SK-N-AS, which do respond to Reelin, do not seem to possess functional receptors. However, very recently Ephrin-B family members have been identified to bind Reelin, and their importance for proper signalling has been demonstrated in the mouse brain (49). We detected high mRNA expression of EphrinB1, EphrinB2 and EphrinB3 in both primary NB and NB cell lines (data not shown), which suggests that additional receptors may be relevant for Reelin signalling in NB. Earlier studies also showed binding of Reelin to $\alpha 3 \beta 1$-integrin and amyloid precursor protein $(23,24,50)$. Taken together, it is very likely that VLDLR and APOER2/ LRP8 are not the only functional receptors and additional molecules influence Reelin signalling in NB.

Reelin in neuroblastoma metastases. Our data suggest that Reelin has a dual function in NB. First, Reelin may act as an inhibitor of migration, when differentiating NB cells produce high amounts of the protein. In this case Reelin may act in an autocrine manner. However, when endogenous Reelin is low, as observed in the majority of NB cell lines and in the advanced tumour stages and high-grade NB cells, the cells may migrate towards a Reelin gradient formed by external sources. To our understanding there are at least two possible sources for Reelin acting in a paracrine mode: firstly, we found Reelin in FCS (data not shown), and it is likely that children may also have high levels of Reelin in their blood, facilitating hematogenic metastases of NB. Secondly, we found Reelin in both tumour BECs and LECs. As we have shown, expression of Reelin in LECs is also found in normal healthy tissues, however, expression in BECs appears to be tumour-specific. Reelin in BECs may promote hematogenic metastases, in LECs it may promote nodal metastases. Both types of metastases can be observed in the clinics of NB. Therefore, loss of Reelin in NB cells may promote both hematogenic and lymphogenic metastases, as tumour cells with low endogenous Reelin expression tend to migrate towards Reelin from external sources. This is supported by studies of Sato et al who found that knock-down of Reelin in pancreatic cancer increases cell motility, invasiveness and substrate-independent colony formation of tumour cells (51). They also found that Reelin is downregulated in pancreatic cancer, as compared to healthy pancreatic tissue. Together, these findings indicate that reduced Reelin expression is characteristic for less differentiated, more malignant tumours. However, conflicting data showing that Reelin is a marker for aggressiveness in prostate cancer, retinoblastoma and oesophageal cancer also exist and indicate a tissue-specific role of Reelin (52-54). However, the exact localization of Reelin in these tumours remains to be studied.

\section{Acknowledgements}

We thank Mrs. S. Schwoch, Mrs. Ch. Zelent, Mr. B. Manshausen and Mr. F. Ludewig for their excellent technical assistance and Drs Frank Berthold, Barbara Hero and Jessica Theissen of the German Neuroblastoma Studies Group (Children's Hospital University Cologne, Cologne, Germany), for providing tumour samples and data. We are grateful to Professors Tom Curran (CHOP, Philadelphia, PA) and E. Förster (UKE, Hamburg, Germany) for providing Reelin plasmid constructs, Reelinexpressing HEK-293 cells and helpful discussions.

\section{References}

1. Castleberry RP: Neuroblastoma. Eur J Cancer 33: 1430-1438, 1997.

2. Maris JM: Recent advances in neuroblastoma. N Engl J Med 362: 2202-2211, 2010.

3. Maris JM,Hogarty MD, Bagatell R and Cohn SL: Neuroblastoma. Lancet 369: 2106-2120, 2007.

4. Brodeur GM: Neuroblastoma: biological insights into a clinical enigma. Nat Rev Cancer 3: 203-216, 2003.

5. Weinstein JL, Katzenstein HM and Cohn SL: Advances in the diagnosis and treatment of neuroblastoma. Oncologist 8: 278-292, 2003.

6. Westermann F and Schwab M: Genetic parameters of neuroblastomas. Cancer Lett 184: 127-147, 2002.

7. Becker J, Erdlenbruch B, Noskova I, et al: Keratoepithelin suppresses the progression of experimental human neuroblastomas. Cancer Res 66: 5314-5321, 2006.

8. Becker J, Pavlakovic H, Ludewig F, et al: Neuroblastoma progression correlates with downregulation of the lymphangiogenesis inhibitor sVEGFR-2. Clin Cancer Res 16: 1431-1441, 2010.

9. Becker J, Volland S, Noskova I, Schramm A, Schweigerer LL and Wilting J: Keratoepithelin reverts the suppression of tissue factor pathway inhibitor 2 by MYCN in human neuroblastoma: a mechanism to inhibit invasion. Int J Oncol 32: 235-240, 2008.

10. Volland S, Kugler W, Schweigerer L, Wilting J and Becker J: Stanniocalcin 2 promotes invasion and is associated with metastatic stages in neuroblastoma. Int J Cancer 125: 2049-2057, 2009.

11. Norgall S, Papoutsi M, Rossler J, Schweigerer L, Wilting J and Weich HA: Elevated expression of VEGFR-3 in lymphatic endothelial cells from lymphangiomas. BMC Cancer 7: 105, 2007.

12. Petrova TV, Makinen T, Makela TP, et al: Lymphatic endothelial reprogramming of vascular endothelial cells by the Prox-1 homeobox transcription factor. EMBO J 21: 4593-4599, 2002.

13. Podgrabinska S, Braun P, Velasco P, Kloos B, Pepper MS and Skobe M: Molecular characterization of lymphatic endothelial cells. Proc Natl Acad Sci USA 99: 16069-16074, 2002.

14. D'Arcangelo G, Miao GG, Chen SC, Soares HD, Morgan JI and Curran T: A protein related to extracellular matrix proteins deleted in the mouse mutant reeler. Nature 374: 719-723, 1995. 
15. Kohno $\mathrm{T}$ and Hattori M: Re-evaluation of protease activity of reelin. Biol Pharm Bull 33: 1047-1049, 2010.

16. Quattrocchi CC, Wannenes F, Persico AM, et al: Reelin is a serine protease of the extracellular matrix. J Biol Chem 277: 303-309, 2002

17. D'Arcangelo G, Homayouni R, Keshvara L, Rice DS, Sheldon M and Curran T: Reelin is a ligand for lipoprotein receptors. Neuron 24: 471-479, 1999.

18. Benhayon D, Magdaleno S and Curran T: Binding of purified Reelin to ApoER2 and VLDLR mediates tyrosine phosphorylation of Disabled-1. Brain Res Mol Brain Res 112: 33-45, 2003.

19. Hiesberger T, Trommsdorff M, Howell BW, et al: Direct binding of Reelin to VLDL receptor and ApoE receptor 2 induces tyrosine phosphorylation of disabled-1 and modulates tau phosphorylation. Neuron 24: 481-489, 1999.

20. Howell BW, Hawkes R, Soriano P and Cooper JA: Neuronal position in the developing brain is regulated by mouse disabled-1. Nature 389: 733-737, 1997.

21. Strasser V, Fasching D, Hauser C, et al: Receptor clustering is involved in Reelin signaling. Mol Cell Biol 24: 1378-1386, 2004

22. Trommsdorff M, Gotthardt M, Hiesberger T, et al: Reeler/ Disabled-like disruption of neuronal migration in knockout mice lacking the VLDL receptor and ApoE receptor 2. Cell 97: 689-701, 1999

23. Dulabon L, Olson EC, Taglienti MG, et al: Reelin binds alpha3 beta1 integrin and inhibits neuronal migration. Neuron 27 33-44, 2000

24. Hoe HS, Lee KJ, Carney RS, et al: Interaction of reelin with amyloid precursor protein promotes neurite outgrowth J Neurosci 29: 7459-7473, 2009.

25. Yip YP, Kronstadt-O'Brien P, Capriotti C, Cooper JA and Yip JW: Migration of sympathetic preganglionic neurons in the spinal cord is regulated by Reelin-dependent Dab1 tyrosine phosphorylation and CrkL. J Comp Neurol 502: 635-643, 2007.

26. Evangelisti C, Florian MC, Massimi I, et al: MiR-128 up-regulation inhibits Reelin and DCX expression and reduces neuroblastoma cell motility and invasiveness. FASEB J 23 4276-4287, 2009.

27. Hughes M, Marsden HB and Palmer MK: Histologic patterns of neuroblastoma related to prognosis and clinical staging. Cance 34: 1706-1711, 1974.

28. Peuchmaur M, d'Amore ES, Joshi VV, et al: Revision of the International Neuroblastoma Pathology Classification: confirmation of favorable and unfavorable prognostic subsets in ganglioneuroblastoma, nodular. Cancer 98: 2274-2281, 2003.

29. Becker J, Wang B, Pavlakovic H, Buttler K and Wilting J: Homeobox transcription factor Prox1 in sympathetic ganglia of vertebrate embryos: correlation with human stage $4 \mathrm{~s}$ neuroblastoma. Pediatr Res 68: 112-117, 2010

30. Chen Y, Kundakovic M, Agis-Balboa RC, Pinna G and Grayson DR: Induction of the reelin promoter by retinoic acid is mediated by Sp1. J Neurochem 103: 650-665, 2007.

31. Pahlman S, Hoehner JC, Nanberg E, et al: Differentiation and survival influences of growth factors in human neuroblastoma. Eur J Cancer 31A: 453-458, 1995.

32. Wilting J, Papoutsi M, Christ B, et al: The transcription factor Prox1 is a marker for lymphatic endothelial cells in normal and diseased human tissues. FASEB J 16: 1271-1273, 2002.

33. Lagodny J, Juttner E, Kayser G, Niemeyer CM and Rossler J: Lymphangiogenesis and its regulation in human neuroblastoma. Biochem Biophys Res Commun 352: 571-577, 2007.

34. Falconer DS and Sierts-Roth U: Dreher, a new gene of the waltzer-shaker group in the house mouse. Z Indukt Abstamm Vererbungsl 84: 71-73, 1951 (In undetermined language).

35. Fatemi SH: Reelin mutations in mouse and man: from reeler mouse to schizophrenia, mood disorders, autism and lissencephaly. Mol Psychiatry 6: 129-133, 2001.
36. Hourihane JO, Bennett CP, Chaudhuri R, Robb SA and Martin ND: A sibship with a neuronal migration defect, cerebellar hypoplasia and congenital lymphedema. Neuropediatrics 24: 43-46, 1993

37. Lorenzetto E, Panteri R, Marino R, Keller F and Buffelli M: Impaired nerve regeneration in reeler mice after peripheral nerve injury. Eur J Neurosci 27: 12-19, 2008.

38. Panteri R, Mey J, Zhelyaznik N, et al: Reelin is transiently expressed in the peripheral nerve during development and is upregulated following nerve crush. Mol Cell Neurosci 32: 133-142, 2006

39. Smalheiser NR, Costa E, Guidotti A, et al: Expression of reelin in adult mammalian blood, liver, pituitary pars intermedia, and adrenal chromaffin cells. Proc Natl Acad Sci USA 97: 1281-1286, 2000.

40. Kim DH, Iijima H, Goto K, et al: Human apolipoprotein E receptor 2. A novel lipoprotein receptor of the low density lipoprotein receptor family predominantly expressed in brain. J Biol Chem 271: 8373-8380, 1996

41. Koch S, Strasser V, Hauser C, et al: A secreted soluble form of ApoE receptor 2 acts as a dominant-negative receptor and inhibits Reelin signaling. EMBO J 21: 5996-6004, 2002.

42. Brandes AA, Palmisano V, Pasetto LM, Basso U and Monfardini S: High-dose chemotherapy with bone marrow rescue for high-grade gliomas in adults. Cancer Invest 19: 41-48, 2001.

43. Brandes C, Novak S, Stockinger W, Herz J, Schneider WJ and Nimpf J: Avian and murine LR8B and human apolipoprotein E receptor 2: differentially spliced products from corresponding genes. Genomics 42: 185-191, 1997.

44. Kim DH, Magoori K, Inoue TR, et al: Exon/intron organization, chromosome localization, alternative splicing, and transcription units of the human apolipoprotein E receptor 2 gene. J Biol Chem 272: 8498-8504, 1997.

45. Bock HH, Jossin Y, Liu P, et al: Phosphatidylinositol 3-kinase interacts with the adaptor protein Dab1 in response to Reelin signaling and is required for normal cortical lamination. J Biol Chem 278: 38772-38779, 2003.

46. Thiele C: Neuroblastoma cell lines. In: Human Cell Culture. Vol 1. Masters J (ed). Kluwer Academic Publishers, Lancaster, pp21-53, 1998.

47. Zhao S and Frotscher M: Go or stop? Divergent roles of Reelin in radial neuronal migration. Neuroscientist 16: 421-434, 2010.

48. Meyer G and Goffinet AM: Prenatal development of reelinimmunoreactive neurons in the human neocortex. J Comp Neurol 397: 29-40, 1998.

49. Senturk A, Pfennig S, Weiss A, Burk K and Acker-Palmer A: Ephrin Bs are essential components of the Reelin pathway to regulate neuronal migration. Nature 472: 356-360, 2011.

50. Schmid RS, Jo R, Shelton S, Kreidberg JA and Anton ES: Reelin, integrin and DAB1 interactions during embryonic cerebral cortical development. Cereb Cortex 15: 1632-1636, 2005.

51. Sato N, Fukushima N, Chang R, Matsubayashi $\mathrm{H}$ and Goggins M: Differential and epigenetic gene expression profiling identifies frequent disruption of the RELN pathway in pancreatic cancers. Gastroenterology 130: 548-565, 2006.

52. Perrone G, Vincenzi B, Zagami M, et al: Reelin expression in human prostate cancer: a marker of tumor aggressiveness based on correlation with grade. Mod Pathol 20: 344-351, 2007.

53. Seigel GM, Hackam AS, Ganguly A, Mandell LM and Gonzalez-Fernandez F: Human embryonic and neuronal stem cell markers in retinoblastoma. Mol Vis 13: 823-832, 2007.

54. Wang Q, Lu J, Yang C, et al: CASK and its target gene Reelin were co-upregulated in human esophageal carcinoma. Cancer Lett 179: 71-77, 2002. 